\title{
Learning Evaluation of Online Micro@Masters Programs of Internalisation at Home: Application of the Upgraded Kirkpatrick's Model
}

\author{
Pedja Ašanin Gole ${ }^{1}$, Ahmet Kadri Kusum², Dejan Andonov³ ${ }^{3}$ M. Adela Gjorgjioska ${ }^{4}$ \\ ${ }^{1}$ DOBA Faculty of Applied Business and Social Studies Maribor, Slovenia \\ ${ }^{2}$ Istanbul University, Istanbul, Turkey \\ ${ }^{3,4}$ Institute of Communication Studies, Skopje, North Macedonia
}

\begin{abstract}
We evaluate internationalisation at home $(\mathrm{IaH})$ as a means for bridging the 'mobility gap' in countries, which are outside the core of Europe. It draws on the experience of two six month long international Micro@ Masters programs, which were conducted jointly within the virtual campus of four higher education institutions (University of Istanbul, Turkey; Institute of Communication Studies, North Macedonia; DOBA Faculty of Applied Business and Social Studies, Slovenia; and Vytautas Magnus University, Lithuania) in the period between 2018 and 2021. We have developed and implemented two six-month online micro master programs: (1) entrepreneurship and innovative strategies, and (2) digital communication and marketing. For both programs we have provided the virtual campus with online learning management system, teaching and learning e-materials, 20 lectures from four countries, 273 students from 9 countries who carried out learning activities within mixed international teams. We used a questionnaire ( $\mathrm{n}=153$ students enrolled in one of the two Micro@Masters programs) and semistructured interviews with lecturers $(n=9)$ and students $(n=17)$. Using the updated Kirkpatrick's model in order to assess the utility and effectiveness of $\mathrm{IaH}$, we concluded that, by effectively delivering IaH, the Micro@Masters programmes have contributed towards addressing the 'mobility imperative' in countries that fall outside of the core of Europe. As a result, they represent the ways in which online education can be used as a tool for bridging the inequality gap in the provision of a more equally distributed internationalised higher education across Europe.
\end{abstract}

Keywords: learner's learning outcomes; learning transferred into practice; mobility gap; online learning experience; ultimate impact of training 